\title{
PURE BARGAINING PROBLEMS WITH A COALITION STRUCTURE*
}

\author{
Francesc Carreras ${ }^{\dagger}$ and Guillermo Owen ${ }^{\ddagger}$
}

May 21, 2016

\begin{abstract}
We consider here pure bargaining problems endowed with a coalition structure such that each union is given its own utility. In this context we use the Shapley rule in order to assess the main options available to the agents: individual behavior, cooperative behavior, isolated unions behavior, and bargaining unions behavior. The latter two respectively recall the treatment given by Aumann-Drèze and Owen to cooperative games with a coalition structure. A numerical example illustrates the procedure. We provide criteria to compare any pair of behaviors for each agent, introduce and axiomatically characterize a modified Shapley rule, and determine its natural domain, that is, the set of problems where the bargaining unions behavior is the best option for all agents.
\end{abstract}

Keywords: pure bargaining problem, coalition structure, Shapley rule. AMS subject classification: 91A12. JEL code: C71.

\section{Introduction}

A consistent alternative to the classical proportional rule, the Shapley rule, has been suggested in $[5]^{1}$ as a much better solution for pure bargaining problems. It derives from the classical Shapley value for cooperative games [11] (also in [10]) because any pure bargaining problem can be linearly identified in a one-to-one way with a quasi-additive cooperative game. We consider here a more complicated setup, that of pure bargaining problems endowed with a coalition structure.

* Research partially supported by Grant MTM 2012-34426 of the Economy and Competitiveness Spanish Ministry and the European Regional Development Fund, which sponsored Owen's visits to Terrassa in May 2014 and April 2015.

$\dagger$ Department of Mathematics and Terrassa School of Industrial, Aerospace and Audiovisual Engineering, Universitat Politècnica de Catalunya (UPC), Spain. E-mail: francesc.carreras@upc.edu. Corresponding author. Mailing address: ESEIAAT, P.O. Box 577, E-08220 Terrassa, Spain.

†Department of Mathematics, Naval Postgraduate School, Monterey, California. E-mail: gowen@nps.edu.

${ }^{1}$ This article was selected by editors M.J. Holler and H. Nurmi to be reproduced in a commemorative volume [6]. We feel honored by this distinction. 
The organization of the paper is as follows. In Section 2 we briefly recall the notions of pure bargaining problem and Shapley rule introduced in [5] and include a new axiomatic characterization of this rule. In Section 3 we consider pure bargaining problems with a coalition structure (CS-problems) and discuss the main options available to the agents: individual behavior (I), cooperative behavior (C), isolated unions behavior (U), and bargaining unions behavior (B). Essentially, the former two are the options in a pure bargaining problem, whereas the latter two respectively recall the treatment given by Aumann-Drèze [3] and Owen [8] (also in [9]) to cooperative games with a coalition structure. A numerical example is presented and discussed in Section 4. In Section 5, a main result characterizes all agents' and unions' preferences on the four options to act. In Section 6 we introduce the modified Shapley rule for pure bargaining problems with a coalition structure, provide an axiomatic characterization of this rule, and determine its natural domain, that is, the set of pure bargaining problems with a coalition structure where the bargaining unions behavior is the best option for all agents. Section 7 concludes.

\section{Pure bargaining problems and the Shapley rule}

Let $N=\{1,2, \ldots, n\}$ (with $n \geq 1$ ) be a set of agents and assume that there are given: (a) a set of utilities $u_{1}, u_{2}, \ldots, u_{n}$ available to the agents individually and (b) a total utility $u_{N}$ that the agents can jointly obtain if all of them agree to cooperate to this end. We assume that utility is transferable, and utilities denoting costs will be represented by negative numbers. ${ }^{2}$ Of course, if $n=1$ then we impose $u_{N}=u_{1}$. A vector $u=\left(u_{1}, u_{2}, \ldots, u_{n} \mid u_{N}\right)$ collects all this information and we will say that it represents a pure bargaining problem (often simply problem, in the sequel) in $N$. An important parameter is the surplus of $u$, defined as

$$
\Delta(u)=u_{N}-\sum_{j \in N} u_{j}
$$

We say that $u$ is additive if $\Delta(u)=0$ and superadditive if $\Delta(u)>0$. The latter is the most interesting case since the problem consists in sharing $u_{N}$ among the agents in a rational way, i.e. in such a manner that all of them should agree and feel (more or less) satisfied with the outcome. Of course, the individual utilities $u_{1}, u_{2}, \ldots, u_{n}$ should be taken into account, so when $\Delta(u)>0$ there is something to gain by cooperating. For shortness we will also say that $u$ is (super)additive if it is either additive or superadditive, i.e. when $\Delta(u) \geq 0$.

Example 2.1 (A cost allocation problem) Assume that three consumers, $A, B$ and $C$, wish to get some kind of supply (electricity, water, gas) from a supplier $S$. The locations are $A(2,2)$, $B(-2,2), C(-2,-2)$ and $S(2,0)$, the distances being given in kilometers (see Fig. 1). The connection costs amount to 100 monetary units per $\mathrm{km}$.

For individual connections, the supplier offers using the dashed lines $S A, S B$ and $S C$. For $A$, $B$ and $C$ together (that is, under a joint contract), the offer consists in using $S A, S O, O B$ and $O C$ (thick line). The question is how to share the joint connection cost. Then we have a (rounded) superadditive cost problem $u=(-200,-447,-447 \mid-966)$ that describes the individual and joint costs and is defined in $N=\{1,2,3\}$, where 1 is $A, 2$ is $B$ and 3 is $C$. Assume that the three consumers sign a joint contract with the supplier. How should they share the total cost of 966 ?

\footnotetext{
${ }^{2}$ This avoids introducing subadditivity as a desirable property for cost problems.
} 


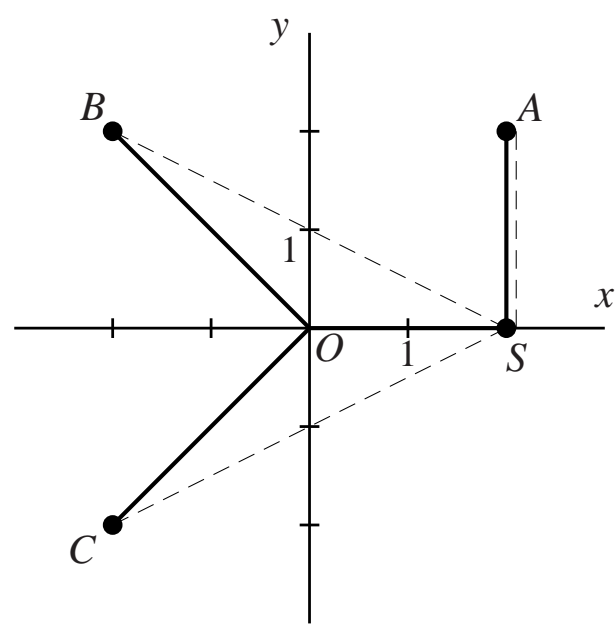

Fig. 1: Locations of consumers and supplier and connections

Let $E_{n+1}=\mathbb{R}^{n} \times \mathbb{R}$ denote the $(n+1)$-dimensional vector space formed by all pure bargaining problems in $N$. In order to deal with, and solve, all these problems, one should look for a sharing rule, i.e. a function $f: E_{n+1} \longrightarrow \mathbb{R}^{n}$. Given $u \in E_{n+1}$, for each $i \in N$ the $i$-coordinate $f_{i}[u]$ of vector $f[u]$ would give the share of $u_{N}$ that is allocated to agent $i$ according to $f$. The proposal made in [5] is the Shapley rule, denoted here as $\varphi$ and defined by

$$
\varphi_{i}[u]=u_{i}+\frac{\Delta(u)}{n} \quad \text { for each } i \in N \text { and each } u \in E_{n+1} .
$$

We refer to [5] for a rationale on this rule and any additional information.

Thus, the Shapley rule solves each problem in the following way: (a) first, each agent is allocated his individual utility; (b) once this has been done, the remaining utility — the surplusis equally shared among all agents. The Shapley rule shows therefore an "egalitarian flavor" in the sense of [4]. Indeed, this rule is a mixture consisting of a "competitive" component, which rewards each agent according to the individual utility, and a "solidarity" component that treats all agents equally. It satisfies standardness for two-agent problems in the sense of [7], and also individual rationality in the (super)additive case since

$$
\varphi_{i}[u] \geq u_{i} \text { for all } i \in N \quad \text { iff } \quad \Delta(u) \geq 0 .
$$

Example 2.2 In Example 2.1, $\Delta(u)=128$ so the Shapley rule saves one third of this to each consumer and yields

$$
\varphi[u]=(-157.33,-404.33,-404.33) .
$$

An axiomatic characterization of the Shapley rule ${ }^{3}$ will close these preliminaries. We omit the proof and the checking of the logical independence of the axiomatic system (cf. Appendix, Subsection 8.1). Let us consider the following properties for a sharing rule $f: E_{n+1} \longrightarrow \mathbb{R}^{n}$.

(i) Group rationality: $\sum_{i \in N} f_{i}[u]=u_{N}$ for every $u \in E_{n+1}$.

(ii) Individual rationality: if $\Delta(u) \geq 0$ then $f_{i}[u] \geq u_{i}$ for all $i \in N$.

\footnotetext{
${ }^{3}$ Alternative characterizations can be found in [5].
} 
(iii) Symmetry: if $u_{i}=u_{j}$ then $f_{i}[u]=f_{j}[u]$.

(iv) Additivity: $f[u+v]=f[u]+f[v]$ for all $u, v \in E_{n+1}$.

Then we have:

Theorem 2.3 There is one and only one sharing rule on $E_{n+1}$ that satisfies properties (i)-(iv). It is the Shapley rule $\varphi$.

Remark 2.4 Let $E_{n+1}^{(s) a}$ be the closed cone formed by all (super)additive problems, that is, problems $u$ such that $\Delta(u) \geq 0$. As was said before, these are the most interesting problems. The cone structure implies that $u+v \in E_{n+1}^{(s) a}$ and $\lambda u \in E_{n+1}^{(s) a}$ for any $u, v \in E_{n+1}^{(s) a}$ and any $\lambda \geq 0$. Thus, property (iv), as well as (i)-(iii), is full of sense in $E_{n+1}^{(s) a}$. Since all members of the basis $\mathcal{B}_{0}$ belong to this cone, the same proof as in Theorem 2.3 shows that the next statement holds:

There is one and only one sharing rule $f: E_{n+1}^{(s) a} \longrightarrow \mathbb{R}^{n}$ that satisfies properties (i)-(iv) in its domain. It is (the restriction to $E_{n+1}^{(s) a}$ of) the Shapley rule $\varphi$.

\section{Pure bargaining problems with a coalition structure}

We consider here a more complicated framework, that of pure bargaining problems endowed with a coalition structure, and discuss the application of the Shapley rule $\varphi$ to this new setup.

The general model is as follows. Let $N=\{1,2, \ldots, n\}$ (with $n \geq 1$ ) be a set of agents and $u=\left(u_{1}, u_{2}, \ldots, u_{n} \mid u_{N}\right)$ be a pure bargaining problem in $N$. Now let us also assume that a coalition structure $B=\left\{B_{1}, B_{2}, \ldots, B_{m}\right\}$ (with $m \geq 1$ ) and utilities $u_{1}^{*}, u_{2}^{*}, \ldots, u_{m}^{*}$ and $u_{M}^{*}$ are given, where $M=\{1,2, \ldots, m\}$ represents the set of unions understood as supra-agents. Each $u_{k}^{*}$ is the utility that the agents of union $B_{k}$ can jointly obtain if all of them cooperate to this end, independently of the remaining agents - those of $N \backslash B_{k}$. If $B_{k}=\{i\}$ we assume that $u_{k}^{*}=u_{i}$. Finally, $u_{M}^{*}$ is the total utility that the unions can jointly obtain by acting as supra-agents. Thus, we do not necessarily assume that $u_{k}^{*}=\sum_{i \in B_{k}} u_{i}$ for each $k$ nor that $u_{M}^{*}=u_{N}$.

Vector $u^{*}=\left(u_{1}^{*}, u_{2}^{*}, \ldots, u_{m}^{*} \mid u_{M}^{*}\right)$ will be called the quotient problem (in $M$ ), and $\left[u, B, u^{*}\right]$ a pure bargaining problem with a coalition structure or, for short, a CS-problem. In the particular case where $u_{M}^{*}=u_{N}$, the model might be viewed as an intermediate step between a pure bargaining problem, where only the individual utilities and the total one are given, and a cooperative game, where a utility $u(S)$ for each $S \subseteq N$ is given.

In order to be coherent with the simple problem model considered in the previous section, for trivial coalition structures we assume that no new information is provided. Hence, if $B=$ $B^{n}=\{\{1\},\{2\}, \ldots,\{n\}\}$ then $M=N$ and $B_{i}=\{i\}$ for each $i \in N$; so, in addition to $u_{i}^{*}=u_{i}$ for all $i$, we impose $u_{M}^{*}=u_{N}$. And if $B=B^{N}=\{N\}$ then $M=\{1\}$ and $B_{1}=N$, and we impose that $u_{1}^{*}=u_{M}^{*}=u_{N}$. Therefore, in both cases $\left[u, B, u^{*}\right]$ essentially reduces to $u$.

In a pure bargaining problem $u$, the agents have only two options: agreeing all together in cooperating to obtain the total utility $u_{N}$ and share it or, otherwise, and even if just one of them disagrees, acting individually and merely getting the individual utilities. Instead, given a CS-problem $\left[u, B, u^{*}\right]$, four main options are available to the agents: 
- Individual behavior (I). The agents decide to act individually and obtain $u_{1}, u_{2}, \ldots, u_{n}$, respectively. This is a sort of "disagreement point" to which they can always go back if the next options are not successful.

- Cooperative behavior (C). All agents agree to cooperate in order to obtain $u_{N}$ and share it using the Shapley rule, disregarding the coalition structure and hence the next possibilities to act via unions.

- Isolated unions behavior (U). All agents of each union $B_{k}$ agree to cooperate in order to obtain $u_{k}^{*}$ and share it using the Shapley rule. (Maybe this will be the behavior only in some unions, in which case the agents of the remaining ones will be forced to act individually.) If $B_{k}=\left\{i_{1}, i_{2}, \ldots, i_{b_{k}}\right\}$, the local problem in $B_{k}$ is

$$
u^{k}=\left(u_{i_{1}}, u_{i_{2}}, \ldots, u_{i_{b_{k}}} \mid u_{k}^{*}\right)
$$

Notice that if $B_{k}=\{i\}$ then $u_{k}^{*}=u_{i}$.

This behavior recalls Aumann and Drèze's approach [3] when discussing the extension of the classical Shapley value to cooperative games with a coalition structure, which leads to a solution that consists in applying the Shapley value to the subgame played in each union. Here, the Shapley rule is applied to each local problem.

- Bargaining unions behavior (B). This is a two-step procedure that requires the agreement of all agents at two levels: forming the union they belong to, and allowing then it to agree with the other unions. Then all unions bargain first among themselves in the quotient problem

$$
u^{*}=\left(u_{1}^{*}, u_{2}^{*}, \ldots, u_{m}^{*} \mid u_{M}^{*}\right)
$$

and share $u_{M}^{*}$ by using the Shapley rule. Thus, each union $B_{k}$ gets $\varphi_{k}\left[u^{*}\right]$. Next, within each $B_{k}$ its agents agree to cooperate for sharing $\varphi_{k}\left[u^{*}\right]$ using again the Shapley rule. If $B_{k}=\left\{i_{1}, i_{2}, \ldots, i_{b_{k}}\right\}$ and $b_{k}>1$, these agents act in the alternative local problem given by

$$
\bar{u}^{k}=\left(u_{i_{1}}, u_{i_{2}}, \ldots, u_{i_{b_{k}}} \mid \varphi_{k}\left[u^{*}\right]\right)
$$

and apply the Shapley rule to this problem. If $b_{k}=1$ the unique agent in $B_{k}$ directly gets $\varphi_{k}\left[u^{*}\right] .{ }^{4}$ This behavior recalls Owen's approach [8] (also in [9]) when introducing the coalition value for cooperative games with a coalition structure: this value consists in applying, first, the Shapley value to the quotient game played by the unions and applying, then, the Shapley value again to an internal game within each union to share among its members the payoff obtained by that union in the quotient game. Here, the procedure is very similar, but using at both levels the Shapley rule.

As expected, for trivial structures these four options reduce to the standard ones in a pure bargaining problem. Indeed, we have that (for all agents): (a) if $B=B^{n}$ then $U$ reduces to I and $\mathrm{B}$ to $\mathrm{C}$; (b) if $B=B^{N}$ then $\mathrm{U}$ and $\mathrm{B}$ reduce to $\mathrm{C}$.

\footnotetext{
${ }^{4}$ In fact, if $B_{k}=\{i\}$ then $\left(u_{i} \mid \varphi_{k}\left[u^{*}\right]\right)$ might not be a pure bargaining problem, but it is clear that, acting as "supra-agent" $k$ representing himself, agent $i$ can obtain $\varphi_{k}\left[u^{*}\right]$ if all unions choose the behavioral option B.
} 


\section{A numerical example}

To see how this model works, we will consider a 9-person CS-problem. (It is difficult to see how a smaller problem would allow us to illustrate this, since we should, as a practical matter, have at least three unions, each with approximately three agents.) We are grateful to Fabrice Valognes, of the Université of Caen-BasseNormandie, for a suggestion he made us.

Example 4.1 (A cost allocation problem with a coalition structure) Suppose that 9 manufacturers, located at points $A, B, C, D, E, F, G, H$, and $J$, need to get some particular supply from a distributor located at point $S$. [Note that the 9 manufacturers are not competitors, as they produce different consumer goods. $]^{5}$ They are located in three different cities, and those in each city have generally good relations, working (perhaps) through the local chambers of commerce, each one of which has legal ability to represent its members jointly and also to negotiate with the other chambers in dealing with the distributor.

The locations of the agents (manufacturers) and the distributor are shown in Fig. 2. We will be interested only in transportation costs (i.e. connection costs) and will assume, in order to make calculations easier, that the cost of connecting any two points is proportional to the square of the distance between them. ${ }^{6}$

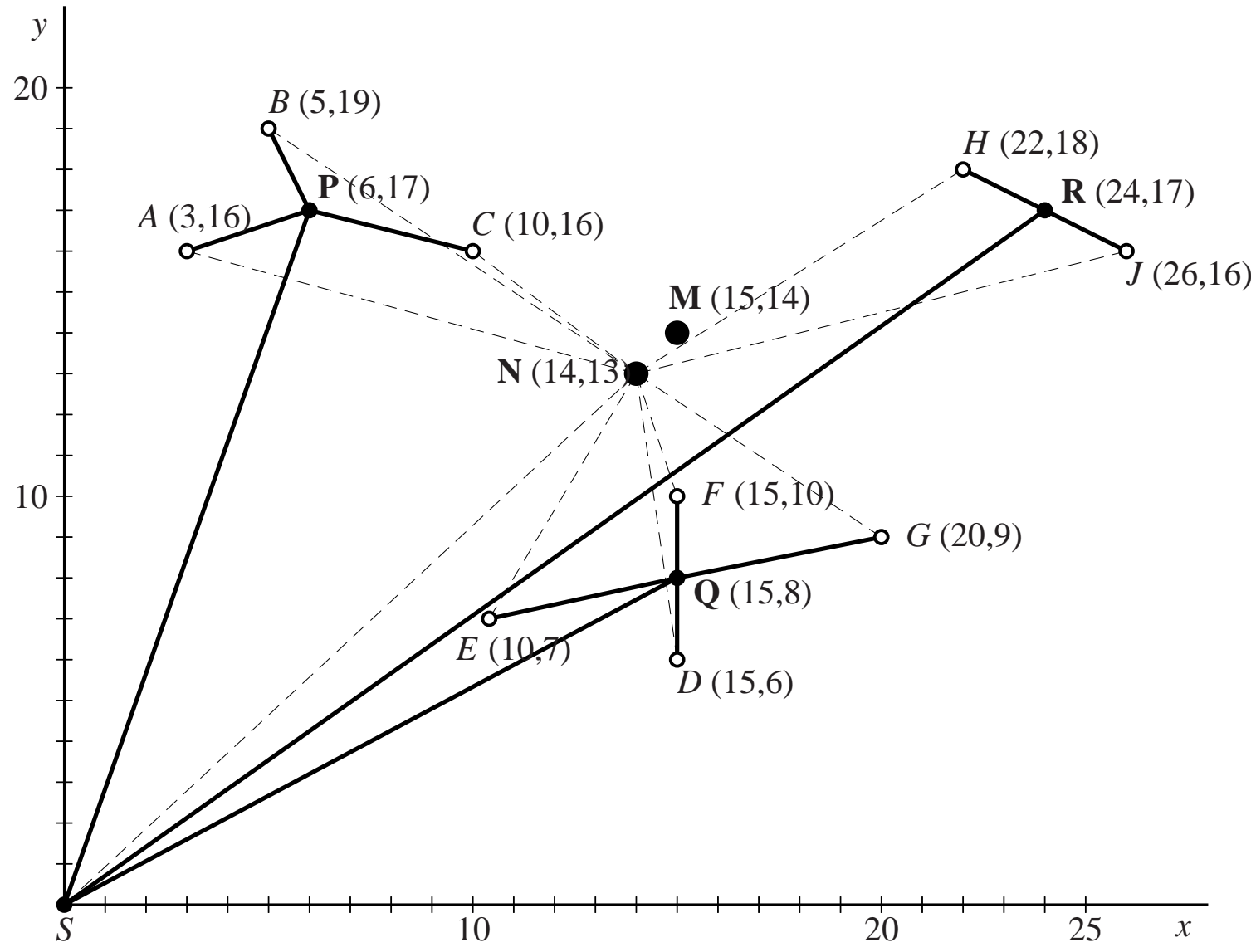

Fig. 2: Locations of manufacturers and distributor, total and local barycenters, and connections

\footnotetext{
${ }^{5}$ E.g. we might assume that the manufacturers produce machines and vehicles of various types and constitute an oligopoly, while the distributor is a monopolist of a raw material such as steel.

${ }^{6}$ Since the Shapley rule is linear and hence homogeneous, and since we are only interested in comparing costs, we may take the factor of proportionality equal to 1 .
} 
If some set of manufacturers sign a joint contract with the distributor to obtain a common connection, the procedure chosen by the distributor will consist in all cases in establishing a connection from $S$ to the barycenter of the locations of these manufacturers and connecting then this barycenter to each manufacturer. Thus, for the cooperative behavior $\mathrm{C}$ we will use the "total" barycenter $\mathbf{N}$ (for the nine manufacturers), the location of which is given in Fig. 2. The dashed lines represent the total connection with all manufacturers via $\mathbf{N}$.

However, the manufacturers are associated in the local chambers of commerce of each city. These are

$$
B_{1}=\{A, B, C\}, \quad B_{2}=\{D, E, F, G\} \quad \text { and } \quad B_{3}=\{H, J\},
$$

and this is the coalition structure $B=\left\{B_{1}, B_{2}, B_{3}\right\}$ that will be considered here. Then, whenever the unions effectively matter, i.e. for the isolated unions behavior $U$ and the bargaining unions behavior B, we will also use the "local" (city) barycenters $\mathbf{P}, \mathbf{Q}$ and $\mathbf{R}$, referred to (the agents of) unions $B_{1}, B_{2}$ and $B_{3}$, respectively. The locations of these three points are also indicated in Fig. 2, and the thick lines represent the local connections using the corresponding barycenters. Besides, we will consider the "unions barycenter" $\mathbf{M}$, the barycenter of $\mathbf{P}, \mathbf{Q}$ and $\mathbf{R}$ also described in Fig. 2, to compute $u_{M}^{*}$, which will be used for the bargaining unions behavior B.

Then we will analyze the cost CS-problem $\left[u, B, u^{*}\right]$ given by

$$
\begin{aligned}
& u=(-265,-386,-356,-261,-149,-325,-481,-808,-932 \mid-1043), \\
& B=\left\{B_{1}, B_{2}, B_{3}\right\}, \quad \text { and } u^{*}=(-357,-349,-875 \mid-739) .
\end{aligned}
$$

where

$$
\begin{aligned}
& u_{M}^{*}=d(S, \mathbf{M})^{2}+\left[d(\mathbf{M}, \mathbf{P})^{2}+\sum_{X \in B_{1}} d(\mathbf{P}, X)^{2}\right]+ \\
& {\left[d(\mathbf{M}, \mathbf{Q})^{2}+\sum_{Y \in B_{2}} d(\mathbf{Q}, Y)^{2}\right]+\left[d(\mathbf{M}, \mathbf{R})^{2}+\sum_{Z \in B_{3}} d(\mathbf{R}, Z)^{2}\right] .}
\end{aligned}
$$

We will consider the four options available to the agents. Table 1 will show the sharing in each case as well as agents' and unions' preferences over the behavior options.

Individual behavior (I). If the manufacturers act all individually, then each one of them signs an individual contract with the distributor and pays the amount indicated by the corresponding component of $u$ : thus, $A$ pays 265, $B$ pays 386, and so on until $J$, who pays 932.

Cooperative behavior (C). If all manufacturers agree to sign a joint contract with the distributor, and collectively pay therefore 1043, the Shapley rule yields the (rounded) sharing of this cost among them:

$$
\varphi[u]=(59.44,-61.56,-31.56,63.44,175.44,-0.56,-156.56,-483.56,-607.56) .
$$

Isolated unions behavior (U). If in each city all manufacturers decide to act together through their chamber of commerce, sign a contract concerning themselves, and disregard therefore the cooperation with the remaining manufacturers, then there are three local problems, to which we will apply the Shapley rule:

$$
\begin{array}{lll}
B_{1}=\{A, B, C\}: & u^{1}=(-265,-386,-356 \mid-357), & \Delta\left(u^{1}\right)=650 \\
B_{2}=\{D, E, F, G\}: & u^{2}=(-261,-149,-325,-481 \mid-349), & \Delta\left(u^{2}\right)=867 \\
B_{3}=\{H, J\}: & u^{3}=(-808,-932 \mid-875), & \Delta\left(u^{3}\right)=865
\end{array}
$$


Bargaining unions behavior (B). The first step is defined by the quotient problem referred to unions:

$$
u^{*}=(-357,-349,-875 \mid-739)
$$

with a surplus $\Delta\left(u^{*}\right)=842$. The Shapley rule yields $\varphi\left[u^{*}\right]=(-76.33,-68.33,-594.33)$. This gives rise to the alternative local problems, where the Shapley rule will be applied again:

$$
\begin{array}{lll}
B_{1}=\{A, B, C\}: & \bar{u}^{1}=(-265,-386,-356 \mid-76.33), & \Delta\left(\bar{u}^{1}\right)=930.67 \\
B_{2}=\{D, E, F, G\}: & \bar{u}^{2}=(-261,-149,-325,-481 \mid-68.33), & \Delta\left(\bar{u}^{2}\right)=1147.67 \\
B_{3}=\{H, J\}: & \bar{u}^{3}=(-808,-932 \mid-1147.67), & \Delta\left(\bar{u}^{3}\right)=1145.67
\end{array}
$$

\begin{tabular}{|l|rrrr|c|}
\hline & $\mathrm{I}$ & $\mathrm{C}$ & $\mathrm{U}$ & $\mathrm{B}$ & preference \\
\hline$A$ & -265.00 & 59.44 & -48.33 & 45.22 & $\mathrm{I}<\mathrm{U}<\mathrm{B}<\mathrm{C}$ \\
$B$ & -386.00 & -61.56 & -169.33 & -75.78 & $\mathrm{I}<\mathrm{U}<\mathrm{B}<\mathrm{C}$ \\
$C$ & -356.00 & -31.56 & -139.33 & -45.78 & $\mathrm{I}<\mathrm{U}<\mathrm{B}<\mathrm{C}$ \\
\hline$B_{1}=\{A, B, C\}$ & -1007.00 & -33.67 & -357.00 & -76.33 & $\mathrm{I}<\mathrm{U}<\mathrm{B}<\mathrm{C}$ \\
\hline$D$ & -261.00 & 63.44 & -44.25 & 25.92 & $\mathrm{I}<\mathrm{U}<\mathrm{B}<\mathrm{C}$ \\
$E$ & -149.00 & 175.44 & 67.75 & 137.92 & $\mathrm{I}<\mathrm{U}<\mathrm{B}<\mathrm{C}$ \\
$F$ & -325.00 & -0.56 & -108.25 & -38.08 & $\mathrm{I}<\mathrm{U}<\mathrm{B}<\mathrm{C}$ \\
$G$ & -481.00 & -156.56 & -264.25 & -194.08 & $\mathrm{I}<\mathrm{U}<\mathrm{B}<\mathrm{C}$ \\
\hline$B_{2}=\{D, E, F, G\}$ & -1216.00 & 81.78 & -349.00 & -68.33 & $\mathrm{I}<\mathrm{U}<\mathrm{B}<\mathrm{C}$ \\
\hline$H$ & -808.00 & -483.56 & -375.50 & -235.17 & $\mathrm{I}<\mathrm{C}<\mathrm{U}<\mathrm{B}$ \\
$J$ & -932.00 & -607.56 & -499.50 & -359.17 & $\mathrm{I}<\mathrm{C}<\mathrm{U}<\mathrm{B}$ \\
\hline$B_{3}=\{H, J\}$ & -1740.00 & -1091.11 & -875.00 & -594.33 & $\mathrm{I}<\mathrm{C}<\mathrm{U}<\mathrm{B}$ \\
\hline$N=B \cup B_{2} \cup B_{3}$ & -3963.00 & -1043.00 & -1581.00 & -739.00 & $\mathrm{I}<\mathrm{U}<\mathrm{C}<\mathrm{B}$ \\
\hline
\end{tabular}

Table 1: Sharing and behavioral preferences

Summing up, the manufacturers' preferences as to the four options are the following:

- $\mathrm{I}<\mathrm{U}<\mathrm{B}<\mathrm{C}$ for $A, B$ and $C$

- $\quad \mathrm{I}<\mathrm{U}<\mathrm{B}<\mathrm{C}$ for $D, E, F$ and $G$

- $\quad \mathrm{I}<\mathrm{C}<\mathrm{U}<\mathrm{B}$ for $H$ and $J$

The conclusion is that, since options $\mathrm{C}$ and $\mathrm{B}$ require the agreement of all agents, they will not be chosen and all unions will follow what we have called the isolated unions behavior (U). 


\section{A general result on preferences}

Some properties of the general model will be established in this section. Most of them are illustrated by Example 4.1. Given a pair of distinct behavioral options (X,Y), any agent will have one and only one preference of the form $\mathrm{X}<\mathrm{Y}, \mathrm{X}=\mathrm{Y}$, or $\mathrm{X}>\mathrm{Y}$, in accordance with the payoffs that the agent obtains under each one of these options. In Theorem 5.1, each possibility will be characterized in terms of surpluses, or surpluses per capita, of the different problems involved in the considered CS-problem.

Since all conditions follow from solving a numerical inequality, the reader should be warned that, to avoid making the statement too cumbersome, we will use $\gtreqless$. This implies that an equivalence like, e.g., $\mathrm{C} \gtreqless \mathrm{I}$ iff $\Delta(u) \gtreqless 0$ will mean that the following conditions hold:

(a) $\mathrm{C}>$ I iff $\Delta(u)>0$.

(b) $\mathrm{C}=\mathrm{I}$ iff $\Delta(u)=0$.

(c) $\mathrm{C}<$ I iff $\Delta(u)<0$.

Given a CS-problem $\left[u, B, u^{*}\right]$, let us recall that $n=|N|, m=|M|$ and $b_{k}=\left|B_{k}\right|$ denote cardinalities, $u=\left(u_{1}, u_{2}, \ldots, u_{n} \mid u_{N}\right)$ and $u^{*}=\left(u_{1}^{*}, u_{2}^{*}, \ldots, u_{m}^{*} \mid u_{M}^{*}\right)$ are the original and quotient problems respectively and, for each $k \in M, u^{k}=\left(u_{i_{1}}, u_{i_{2}}, \ldots, u_{i_{b_{k}}} \mid u_{k}^{*}\right)$ is the local problem in union $B_{k}$ for option $\mathrm{U}$.

Theorem 5.1 Let $\left[u, B, u^{*}\right]$ be a CS-problem in $N$. Then:

(a) $C \gtreqless I$ for all $i \in N$ iff $\Delta(u) \gtreqless 0$.

(b) $U \gtreqless I$ for all $i \in B_{k}$ iff $\Delta\left(u^{k}\right) \gtreqless 0$.

(c) $B \gtreqless I$ for all $i \in B_{k} \quad$ iff $\Delta\left(u^{k}\right)+\frac{\Delta\left(u^{*}\right)}{m} \gtreqless 0$.

(d) $U \gtreqless C$ for all $i \in B_{k} \quad$ iff $\frac{\Delta\left(u^{k}\right)}{b_{k}} \gtreqless \frac{\Delta(u)}{n}$.

(e) $B \gtreqless C$ for all $i \in B_{k} \quad$ iff $\frac{\Delta\left(u^{k}\right)}{b_{k}}+\frac{\Delta\left(u^{*}\right)}{m b_{k}} \gtreqless \frac{\Delta(u)}{n}$.

(f) $B \gtreqless U$ for all $i \in N \quad$ iff $\Delta\left(u^{*}\right) \gtreqless 0$.

Proof. Let $\pi_{i}(\mathrm{X})$ denote the payoff obtained by agent $i$ under the behavioral option $\mathrm{X}$. Then all equivalences follow at once from comparing the expressions of this payoff in each case:

- $\pi_{i}(\mathrm{I})=u_{i}$ for all $i \in N$.

- $\pi_{i}(\mathrm{C})=u_{i}+\frac{\Delta(u)}{n}$ for all $i \in N$. 
- $\pi_{i}(\mathrm{U})=u_{i}+\frac{\Delta\left(u^{k}\right)}{b_{k}}$ for all $i \in B_{k}$.

- $\pi_{i}(\mathrm{~B})=u_{i}+\frac{\Delta\left(u^{k}\right)}{b_{k}}+\frac{\Delta\left(u^{*}\right)}{m b_{k}}$ for all $i \in B_{k}$.

Remark 5.2 We collect here a series of comments and consequences of Theorem 5.1. The first six are illustrated by Example 4.1. The final two refer to what happens if some very special conditions hold.

- Any preference on (I,C) is common for all agents $i \in N$ and depends only on $u$.

- The same happens for (U,B), and the preference depends only on the quotient $u^{*}$.

- The remaining four preferences, i.e. on $(\mathrm{I}, \mathrm{U}),(\mathrm{I}, \mathrm{B}),(\mathrm{C}, \mathrm{U})$ and $(\mathrm{C}, \mathrm{B})$, are common for at least all agents of each union $B_{k}$. By combining this with the previous items it follows that all agents of each union $B_{k}$ order the four options equally, so we can speak of "unions' preferences".

- If $u$ and $u^{*}$ are (super)additive, then $\mathrm{C}$ for all agents, as well as $\mathrm{U}$ and $\mathrm{B}$ for all unions $B_{k}$ such that $u^{k}$ is also (super)additive, are individually rational, in the sense that $\pi_{i}(\mathrm{X}) \geq u_{i}$ for all involved agents $i$ whenever $\mathrm{X}$ is $\mathrm{C}, \mathrm{U}$ or $\mathrm{B}$.

- If $u$ and $u^{*}$ are superadditive, then $\mathrm{C}>\mathrm{I}$ and $\mathrm{B}>\mathrm{U}$ for all agents.

- If, moreover, $u^{k}$ is superadditive for some $k \in M$, then $\mathrm{U}>\mathrm{I}$ and thus $\mathrm{B}>\mathrm{U}>\mathrm{I}$ for all $i \in B_{k}$, and it only remains to place $\mathrm{C}$ within this ordering: as $\mathrm{C}>\mathrm{I}$, five possibilities arise, two of which can be found in Example 4.1.

- If $u_{k}^{*}=\sum_{i \in B_{k}} u_{i}$ for some $k \in M$, then $u^{k}$ is additive and therefore $\mathrm{U}=\mathrm{I}$ for all $i \in B_{k}$.

- If $u_{M}^{*}=u_{N}$ then not all agents can have a common strict preference on $(\mathrm{C}, \mathrm{B})$, and hence only options I and $\mathrm{U}$, or a mixture of them (U for some unions and I for the remaining agents), will be feasible unless $\mathrm{C}=\mathrm{B}$ holds for all agents. This follows from the fact that

$$
\sum_{i \in N} \pi_{i}(C)=u_{N}=u_{M}^{*}=\sum_{k \in M} \varphi_{k}\left[u^{*}\right]=\sum_{k \in M} \sum_{i \in B_{k}} \pi_{i}(B)=\sum_{i \in N} \pi_{i}(B) .
$$

\section{The modified Shapley rule and its natural domain}

Let $N=\{1,2, \ldots, n\}$ be the set of agents, with $n \geq 1$. The set of all CS-problems defined in $N$ is denoted as $E_{N}$, and a coalitional sharing rule on $E_{N}$ means a map $g: E_{N} \longrightarrow \mathbb{R}^{n}$. Given a CS-problem $\left[u, B, u^{*}\right]$ in $N$, for each $i \in N$ the $i$-coordinate $g_{i}\left[u, B, u^{*}\right]$ of vector $g\left[u, B, u^{*}\right]$ gives the utility that is allocated to agent $i$ according to $g$.

Definition 6.1 The modified Shapley rule, denoted by $\psi$, is the coalitional sharing rule on $E_{N}$ that allocates utilities, to all agents of a CS-problem $\left[u, B, u^{*}\right]$ in $N$, according to the bargaining 
unions behavior B. An explicit expression for $\psi$ has been found in the proof of Theorem 5.1, where the allocations were denoted as $\pi_{i}(\mathrm{~B})$ : given $\left[u, B, u^{*}\right]$ in $N$, if $i \in B_{k}$ then

$$
\psi_{i}\left[u, B, u^{*}\right]=u_{i}+\frac{\Delta\left(u^{k}\right)}{b_{k}}+\frac{\Delta\left(u^{*}\right)}{m b_{k}} .
$$

Some first properties of $\psi$ are stated in the following result.

Proposition 6.2 The modified Shapley rule satisfies the following elementary properties:

(a) Trivial coalition structures: if $B=B^{n}$ or $B=B^{N}$ then $\psi\left[u, B, u^{*}\right]=\varphi[u]$.

(b) General coalition structure: for any $B, \quad \sum_{i \in B_{k}} \psi_{i}\left[u, B, u^{*}\right]=\varphi_{k}\left[u^{*}\right]$ for all $k \in M$.

(c) Coalitional symmetry: if $u_{k}^{*}=u_{h}^{*}$ then $\sum_{i \in B_{k}} \psi_{i}\left[u, B, u^{*}\right]=\sum_{j \in B_{h}} \psi_{j}\left[u, B, u^{*}\right]$.

Proof. (a) If $B=B^{n}$ then $M=N$ and $u^{*}=u$, so the first step of the bargaining unions behavior is nothing but the application of $\varphi$ to $u$, and the second one is trivial and does not change anything. If $B=B^{N}$ then $M=\{1\}, B_{1}=N$ and $u^{1}=u$, so in this case the first step is trivial and the second is nothing but the application of $\varphi$ to $u$.

(b) According to the procedure used in the bargaining unions behavior, what is shared among the agents of each union $B_{k}$ is precisely $\varphi_{k}\left[u^{*}\right]$, even if $B_{k}$ is a singleton.

(c) This is an immediate consequence of (b).

In order to characterize axiomatically the modified Shapley rule, let us consider the following properties for a coalitional sharing rule $g: E_{N} \longrightarrow \mathbb{R}^{n}$.

(i) Group rationality: $\sum_{i \in N} g_{i}\left[u, B, u^{*}\right]=u_{M}^{*}$ for every $\left[u, B, u^{*}\right]$ in $N$.

(ii) Individual rationality: if $\Delta\left(u^{*}\right) \geq 0$ and $\Delta\left(u^{k}\right) \geq 0$ for all $k \in M$ then $g_{i}\left[u, B, u^{*}\right] \geq u_{i}$ for all $i \in N$.

(iii) Coalitional rationality: if $\Delta\left(u^{*}\right) \geq 0$ then $\sum_{i \in B_{k}} g_{i}\left[u, B, u^{*}\right] \geq u_{k}^{*}$ for all $k \in M$.

(iv) Symmetry: if $u_{i}=u_{j}$ and $i, j \in B_{k}$ for some $k \in M$ then $g_{i}\left[u, B, u^{*}\right]=g_{j}\left[u, B, u^{*}\right]$.

(v) Additivity: for all $B$ and all $\left[u, B, u^{*}\right]$ and $\left[v, B, v^{*}\right]$ in $E_{N}$,

$$
g\left[u+v, B, u^{*}+v^{*}\right]=g\left[u, B, u^{*}\right]+g\left[v, B, v^{*}\right] .
$$

(vi) Singletons: if $B_{k}=\{i\}$ then $g_{i}\left[u, B, u^{*}\right]=\varphi_{k}\left[u^{*}\right]^{7}$

This set of properties characterizes the modified Shapley rule $\psi$, defined by Eq. (2). The statement is as follows.

\footnotetext{
${ }^{7}$ In Remark 6.8 we will write this equation in an alternative form that avoids mentioning the Shapley rule.
} 
Theorem 6.3 There is one and only one coalitional sharing rule on $E_{N}$ that satisfies properties (i)-(vi). It is the modified Shapley rule $\psi$.

We omit the proof and the checking of the logical independence of the axiomatic system (cf. Appendix, Subsections 8.2 and 8.3). The proof is similar to that of Theorem 2.3. The existence part is straightforward using from Eq. (2), whereas the uniqueness part needs a suitable basis $\mathcal{B}_{0}$ of $E_{N}^{B}$, the vector space of all CS-problems in $N$ with a fixed coalition structure $B$.

Now we are mainly interested in those CS-problems where the bargaining unions behavior $\mathrm{B}$ is the best option for all agents. These problems constitute the natural domain of the modified Shapley rule $\psi$. The basic question is: under which conditions will all agents prefer B to I, C and $\mathrm{U}$, in a given CS-problem $\left[u, B, u^{*}\right]$ in $N$ ? If $B=B^{n}$ then $\mathrm{B}=\mathrm{C}>\mathrm{U}=\mathrm{I}$ iff $\Delta(u)>0$. If $B=B^{N}$ then $\mathrm{B}=\mathrm{U}=\mathrm{C}>\mathrm{I}$ iff $\Delta(u)>0$. For any other coalition structure $B$, since $\mathrm{B}$ requires more agreements than the other three options, we will consider strict preferences only. ${ }^{8}$

Theorem 6.4 Let $\left[u, B, u^{*}\right]$ be any $C S$-problem in $N$ with a nontrivial $B$. Then $B>I, B>C$ and $B>U$ for all agents in $\left[u, B, u^{*}\right]$ iff the following conditions simultaneously hold:

(i) $\Delta\left(u^{k}\right)+\frac{\Delta\left(u^{*}\right)}{m}>0$ for all $k$

(ii) $\frac{\Delta\left(u^{k}\right)}{b_{k}}+\frac{\Delta\left(u^{*}\right)}{m b_{k}}>\frac{\Delta(u)}{n}$ for all $k$

(iii) $\Delta\left(u^{*}\right)>0$

Proof. Parts (c), (e) and (f) of Theorem 5.1 yield these necessary and sufficient conditions.

Remark 6.5 We remark that the superadditivity of $u$ is convenient but not needed, and also that the above conditions (i)-(iii) do not depend on the alternative local problems $\bar{u}^{k}$ used for sharing under the bargaining unions behavior B.

Example 6.6 In particular, any CS-problem $\left[u, B, u^{*}\right]$ such that

(1) $u^{*}$ is superadditive, i.e. $\Delta\left(u^{*}\right)>0$

(2) all $u^{k}$ are (super)additive, i.e. $\Delta\left(u^{k}\right) \geq 0$, and

(3) $\Delta\left(u^{*}\right)$ is large enough in the sense that $\Delta\left(u^{*}\right)>\frac{m b_{k}}{n} \Delta(u)$ for all $k$

satisfies conditions (i)-(iii) of Theorem 6.4. Conditions (1)-(3) are only sufficient but quite reasonable restrictions to obtain that $\mathrm{B}$ is the best option for all agents. Under (1) and (2), condition (3) can be dropped if $u$ is not superadditive, or otherwise it needs only to be checked for the largest union (maximum $b_{k}$ ).

\footnotetext{
${ }^{8}$ For example, it is clear that, in any problem $u$, all agents prefer $\mathrm{C}$ to I $(\mathrm{C}>\mathrm{I})$ iff $u$ is superadditive, i.e. $\Delta(u)>0$. We might include the case where $u$ is additive, i.e. $\Delta(u)=0$, but then $\mathrm{C}=\mathrm{I}$ for all agents and very probably they would choose I since this behavior does not require any agreement. This follows directly or from Theorem 5.1(a).
} 
Thus, for any CS-problem $\left[u, B, u^{*}\right]$ such that B is the best option for all agents, the modified Shapley rule $\psi$ is the natural way to share $u_{M}^{*}$ among all agents. There are two possibilities: following the two-step procedure detailed in Section 3 or applying Eq. (2) directly.

Example 6.7 Let $N=\{1,2,3,4,5,6\}$ (so $n=6$ ) and

$$
u=(5,4,4,2,1,3 \mid 25) .
$$

Let $B=\left\{B_{1}, B_{2}, B_{3}\right\}$, with $B_{1}=\{1,2,3\}, B_{2}=\{4,5\}$ and $B_{3}=\{6\}$. Thus, $b_{1}=3, b_{2}=2$ and $b_{3}=1$. Finally, let

$$
u^{*}=(19,5,3 \mid 45)
$$

be the quotient problem in $M=\{1,2,3\}$, that represents the set of unions as entities (so $m=3$ ). Then $\left[u, B, u^{*}\right]$ is a CS-problem in $N$. The local problems are

$$
u^{1}=(5,4,4 \mid 19), \quad u^{2}=(2,1 \mid 5) \quad \text { and } \quad u^{3}=(3 \mid 3) .
$$

Then, the sufficient conditions of Example 6.6 are satisfied and we conclude that option $B$ is the best behavior in this $C S$-problem for all agents $i \in N .{ }^{9}$ The application of the modified Shapley rule, using for example Eq. (2) and

$$
\Delta\left(u^{1}\right)=6, \quad \Delta\left(u^{2}\right)=2, \quad \Delta\left(u^{3}\right)=0, \quad \text { and } \quad \Delta\left(u^{*}\right)=18
$$

yields

$$
\psi\left[u, B, u^{*}\right]=(9,8,8,6,5,9),
$$

a sharing that satisfy all agents and more than any other behavioral option.

Remark 6.8 The characterization of the modified Shapley rule $\psi$ given by Theorem 6.3 refers, in principle, to $E_{N}$. But, if we define coalitional sharing rule as any map

$$
g: E=\bigcup_{n=1}^{\infty} E_{N} \longrightarrow \bigcup_{n=1}^{\infty} \mathbb{R}^{n}
$$

such that if $\left[u, B, u^{*}\right] \in E_{N}$ then $g\left[u, B, u^{*}\right] \in \mathbb{R}^{n}$, where $n=|N|$, then the axiomatic system makes sense and Theorem 6.3 also holds for $E$. We will use the same symbols $g$ and $\psi$ for all domains on which they are considered.

Then, having in mind this extension, we see that, for any $\left[u, B, u^{*}\right]$ in a given $N$, we can write in $M$, by Proposition 6.2(a),

$$
\psi\left[u^{*}, B^{m}, u^{* *}\right]=\varphi\left[u^{*}\right] .
$$

The singletons property (vi) may then be written as follows:

(vi) Singletons: if $B_{k}=\{i\}$ then $g_{i}\left[u, B, u^{*}\right]=g_{k}\left[u^{*}, B^{m}, u^{* *}\right]$,

which avoids mentioning the Shapley rule.

\footnotetext{
${ }^{9}$ However, notice that the preferences on the remaining three options are not common. Indeed, we find $\mathrm{B}>\mathrm{U}>\mathrm{C}>\mathrm{I}$ for $B_{1}, \quad \mathrm{~B}>\mathrm{U}=\mathrm{C}>\mathrm{I}$ for $B_{2}, \quad$ and $\mathrm{B}>\mathrm{C}>\mathrm{U}=\mathrm{I}$ for $B_{3}$.
} 
Remark 6.9 Let $E^{*}, E_{N}^{*}$ and $\left(E_{N}^{B}\right)^{*}$ be the respective subsets of $E, E_{N}$ and $E_{N}^{B}$ formed by the CS-problems where B is a better option than U, C and I for all agents, i.e. CS-problems that satisfy the conditions of Theorem 6.4. $\left(E_{N}^{B}\right)^{*}$ is an open cone, and Theorem 6.3 gives rise to an axiomatic characterization of the modified Shapley rule on this cone and hence on $E_{N}^{*}$ and $E^{*}$.

Indeed, we have:

Theorem 6.10 There is one and only one coalitional sharing rule defined on $\left(E_{N}^{B}\right)^{*}$ that satisfies properties (i)-(vi) in this cone. It is (the restriction of) the modified Shapley rule $\psi$.

We omit the proof (cf. Appendix, Subsection 8.3).

\section{Conclusions}

Pure bargaining problems, introduced in [5], constitute a natural setup and, at the same time, a simple case of both Nash's bargaining model and the cooperative game model (as they can be identified with quasi-additive games). Their simplicity allows us to better capture the meaning of certain notions, most of which are translated from the cooperative game theory.

The Shapley rule, also introduced in [5], is a well founded solution notion for any pure bargaining problem, which enjoys satisfactory properties similar to those of the Shapley value. It can be clearly distinguished from other previous notions like e.g. some proportionality rules including the classical proportional rule.

The introduction of a coalition structure in a pure bargaining problem by means of the general model presented here constitutes a novelty in the literature. Four behavioral options for the agents of any CS-problem are suggested: two of them (I and C) are equivalent to agents' options in a pure bargaining problem, while the other two ( $U$ and $B$ ) respectively recall the treatment given by Aumann-Drèze and Owen to cooperative games with a coalition structure. ${ }^{10}$ The Shapley rule is intensively used for evaluating the results of each option and gives rise to agents' individual preferences on the issues. A numerical example illustrates all these ideas.

A main result gives complete and easy conditions (eighteen in all) to determine all agents' preferences on I, C, U and B. They are stated in terms of surpluses and surpluses per capita. Finally, a modified Shapley rule for all pure bargaining problems with a coalition structure has been defined and axiomatically characterized for all CS-problems, and even on its natural domain: the cone of CS-problems where the bargaining unions behavior B is the best option for all agents, which has been described previously.

Future work on this research line should focus on: (a) the possibility of considering negotiations restricted to some of the unions; (b) a refinement of our analysis when not all unions have a common best option; (c) the importance of the quotient problem, exogenously given; and (d) the possibility of speaking, with this model, of endogenous coalition formation and discussing the stability of coalition structures (in the sense of a Nash strong equilibrium).

\footnotetext{
${ }^{10}$ Both approaches are currently interesting topics: see e.g. recent references [1] and [2], where the proportional rule and the Shapley value are combined for monotonic games with a coalition structure following, respectively, Owen's and Aumann-Drèze's viewpoint.
} 


\section{Acknowledgements}

The authors wish to thank the editor and two reviewers for their helpful comments on a first draft of the paper.

\section{References}

[1] Alonso-Meijide, J.M. and Carreras, F. [2011]: “The proportional coalitional Shapley value." Expert Systems with Applications 38, 6967-6979.

[2] Alonso-Meijide, J.M., Carreras, F., Costa, J. and García-Jurado, I. [2015]: “The proportional partitional Shapley value." Discrete Applied Mathematics 187, 1-11.

[3] Aumann, R.J. and Drèze, J. [1974]: “Cooperative games with coalition structures.” International Journal of Game Theory 3, 217-237.

[4] Brink, R. van den and Funaki, Y. [2009]: "Axiomatizations of a class of equal surplus sharing solutions for TU-games." Theory and Decision 67, 303-340.

[5] Carreras, F. and Owen, G. [2011]: "Pure bargaining problems and the Shapley rule." Homo Oeconomicus 28, 379-404.

[6] Carreras, F. and Owen, G. [2013]: "Pure bargaining problems and the Shapley rule." In: Power, Voting, and Voting Power: 30 Years After (M.J. Holler and H. Nurmi, eds.), Springer-Verlag, 681-702.

[7] Hart, S. and Mas-Colell, A. [1988]: “The potential of the Shapley value.” In: The Shapley Value: Essays in Honor of Lloyd S. Shapley (A.E. Roth, ed.), Cambridge University Press, $127-137$.

[8] Owen, G. [1977]: "Values of games with a priori unions.” In: Mathematical Economics and Game Theory (R. Henn and O. Moeschlin, eds.), Springer, 76-88.

[9] Owen, G. [2013]: Game Theory. Fourth Ed. in Emerald Group Publishing Limited.

[10] Roth, A.E. (ed.) [1988]: The Shapley Value: Essays in Honor of Lloyd S. Shapley. Cambridge University Press.

[11] Shapley, L.S. [1953]: “A value for $n$-person games.” Annals of Mathematical Studies 28, 307-317. 


\section{Appendix}

Here we collect additional information that might disturb the reading of the article: mainly, detailed proofs of some results.

\subsection{Axiomatic characterization of the Shapley rule}

Let us consider the following properties for a sharing rule $f: E_{n+1} \longrightarrow \mathbb{R}^{n}$.

(i) Group rationality: $\sum_{i \in N} f_{i}[u]=u_{N}$ for every $u \in E_{n+1}$.

(ii) Individual rationality: if $\Delta(u) \geq 0$ then $f_{i}[u] \geq u_{i}$ for all $i \in N$.

(iii) Symmetry: if $u_{i}=u_{j}$ then $f_{i}[u]=f_{j}[u]$.

(iv) Additivity: $f[u+v]=f[u]+f[v]$ for all $u, v \in E_{n+1}$.

We shall show that this set of properties characterizes the Shapley rule $\varphi$, defined by Eq. (1). We will assume in this section that $n \geq 2$, since for $n=1$ we find that, exceptionally, $\operatorname{dim} E_{n+1}=1$, and hence group rationality suffices to characterize $\varphi$.

The following lemma, whose proof is straightforward, will be applied.

Lemma 8.1 (a) A basis $\mathcal{B}_{0}=\left\{u_{0}^{1}, u_{0}^{2}, \ldots, u_{0}^{n}, u_{0}^{N}\right\}$ of $E_{n+1}$ is given by

- $u_{0}^{k}=(1, \ldots, 1,2,1, \ldots, 1 \mid n+1)$ for $k=1,2, \ldots, n$, and

- $u_{0}^{N}=(1,1, \ldots, 1 \mid n+1)$

(b) All $u_{0}^{k}$ for $k=1,2, \ldots, n$ are additive, whereas $u_{0}^{N}$ is superadditive.

(c) Moreover, if $u=\left(u_{1}, u_{2}, \ldots, u_{n} \mid u_{N}\right) \in E_{n+1}$ then it can be uniquely written as a linear combination of the members of $\mathcal{B}_{0}$ as

$$
u=\sum_{k=1}^{n}\left(u_{k}-\frac{u_{N}}{n+1}\right) u_{0}^{k}+\Delta(u) u_{0}^{N} .
$$

Then we have:

Theorem 8.2 (Theorem 2.3) There is one and only one sharing rule on $E_{n+1}$ that satisfies properties (i)-(iv). It is the Shapley rule $\varphi$.

Proof. (Existence) It suffices to check that the Shapley rule satisfies (i)-(iv), and this follows at once from Eq. (1).

(Uniqueness) We shall see that if $f$ satisfies (i)-(iv) then $f=\varphi$. To this end, we will use the basis $\mathcal{B}_{0}$. For any $\lambda \in \mathbb{R}$, from (i) and (ii) it follows that, for any $i \in N$ and $k=1,2, \ldots, n$,

$$
f_{i}\left[\lambda u_{0}^{k}\right]= \begin{cases}2 \lambda & \text { if } i=k \\ \lambda & \text { if } i \neq k\end{cases}
$$


and, from (i) and (iii),

$$
f_{i}\left[\lambda u_{0}^{N}\right]=\lambda \frac{n+1}{n} \quad \text { for all } i \in N
$$

Then, for any $i \in N$ and any $u \in E_{n+1}$, from (iv) and using Eq. (3) we obtain

$$
f_{i}[u]=2\left(u_{i}-\frac{u_{N}}{n+1}\right)+\sum_{k \neq i}\left(u_{k}-\frac{u_{N}}{n+1}\right)+\Delta(u) \frac{n+1}{n}=u_{i}+\frac{\Delta(u)}{n}=\varphi_{i}[u],
$$

so $f=\varphi$.

As to the logical independence of the axiomatic system described above, it suffices to find four rules that satisfy all axioms but one. Only a problem that shows the failure is needed in each case (a counterexample). All counterexamples are (super)additive and all of them might be extended to any number $n$ of agents by adding $n-2$ null agents.

- A rule that fails to satisfy (i) Group rationality.

$$
f_{i}[u]=u_{i} \quad \text { for all } u \in E_{n+1} \text { and all } i \in N .
$$

It is easy to verify that $f$ satisfies (ii), (iii) and (iv). Instead, for $u=(1,1 \mid 3)$ we find that

$$
f_{1}[u]+f_{2}[u]=2 \neq 3=u_{N} .
$$

- A rule that fails to satisfy (ii) Individual rationality.

$$
f_{i}[u]=\frac{u_{N}}{n} \quad \text { for all } u \in E_{n+1} \text { and all } i \in N .
$$

It is easy to verify that $f$ satisfies (i), (iii) and (iv). Instead, for $u=(1,3 \mid 4)$, where $\Delta(u)=0$, we find that

$$
f_{2}[u]=2 \not 3=u_{2} .
$$

- A rule that fails to satisfy (iii) Symmetry.

$$
f_{i}[u]= \begin{cases}u_{1}+\Delta(u) & \text { if } i=1 \\ u_{i} & \text { otherwise }\end{cases}
$$

for all $u \in E_{n+1}$ and all $i \in N$.

It is easy to verify that $f$ satisfies (i), (ii) and (iv). Instead, for $u=(1,1 \mid 3)$ we find that

$$
u_{1}=u_{2} \quad \text { but } \quad f_{1}[u]=2 \neq 1=f_{2}[u] .
$$

- A rule that fails to satisfy (iv) Additivity. It combines proportional rule and Shapley rule. ${ }^{11}$

$$
f_{i}[u]= \begin{cases}\frac{u_{i}}{\sum u_{j}} u_{N} & \text { if } u_{j}>0 \text { for all } j \in N \\ u_{i}+\frac{\Delta(u)}{n} & \text { otherwise }\end{cases}
$$

\footnotetext{
${ }^{11}$ Note that $\frac{u_{i}}{\sum u_{j}} u_{N}=u_{i}+\frac{u_{i}}{\sum u_{j}} \Delta(u)$ provided that $\sum u_{j} \neq 0$ and, in particular, when all $u_{j}>0$.
} 
for all $u \in E_{n+1}$ and all $i \in N$.

It is easy to verify that $f$ satisfies (i), (ii) and (iii). Instead, for $u=(1,2 \mid 9)$ and $v=(0,1 \mid 3)$ we find $u+v=(1,3 \mid 12)$ and

$$
f[u+v]=(5,7) \neq(3,6)+(1,2)=f[u]+f[v] .
$$

\subsection{The domain of all CS-problems}

Let $N=\{1,2, \ldots, n\}$ be the set of agents, with $n \geq 1$. A CS-problem $\left[u, B, u^{*}\right]$ in $N$ is defined by three objects:

- $u=\left(u_{1}, u_{2}, \ldots, u_{n} \mid u_{N}\right)$, which is a problem in $N$

- $B=\left\{B_{1}, B_{2}, \ldots, B_{m}\right\}$, which is a CS in $N$, with $M=\{1,2, \ldots, m\}$ and $m \geq 1$

- $u^{*}=\left(u_{1}^{*}, u_{2}^{*}, \ldots, u_{m}^{*} \mid u_{M}^{*}\right)$, which is a problem in $M$

with the following restrictions:

(r1) If $B=B^{n}$ or $B=B^{N}$ then $u_{M}^{*}=u_{N}$

(r2) if $n=1$ then $u_{N}=u_{1}$

(r3) if $m=1$ then $u_{M}^{*}=u_{1}^{*}$

(r4) if $b_{k}=1$ for some $k \in M$, i.e. if $B_{k}=\{i\}$ is a singleton, then $u_{k}^{*}=u_{i}$

$E_{N}$ will denote the set of all CS-problems defined in $N$, and we put $E=\bigcup_{n=1}^{\infty} E_{N}$. These two sets do not have any structure. Instead, the set $E_{N}^{B}$, formed by all CS-problems defined in $N$ with a fixed coalition structure $B$, becomes a vector space under the natural linear operations given by

- $\left[u, B, u^{*}\right]+\left[v, B, v^{*}\right]=\left[u+v, B, u^{*}+v^{*}\right]$

- $\lambda\left[u, B, u^{*}\right]=\left[\lambda u, B, \lambda u^{*}\right] \quad$ for all $\lambda \in \mathbb{R}$

Thus, having $B$ in mind, any vector of $E_{N}^{B}$ may be understood simply as a pair of problems $u$ and $u^{*}$, a pair that we will denote as $u \odot u^{*}$ when working within $E_{N}^{B}$ for a given $B$.

Theorem 8.3 The dimension of $E_{N}^{B}$ is as follows:

(a) If $n=1$ then $\operatorname{dim} E_{N}^{B}=1$.

(b) If $n>1$ and $m=1$ then $\operatorname{dim} E_{N}^{B}=n+1$.

(c) If $n>1$ and $m=n$ then $\operatorname{dim} E_{N}^{B}=n+1$.

(d) If $n>1$ and $1<m<n$ then $\operatorname{dim} E_{N}^{B}=n+m-p+2$, where $p$ is the number of unions in $B$ that are singletons $(0 \leq p<m)$. 
Proof. At first glance, any vector $u \odot u^{*}$ requires $n+1+m+1$ linear parameters to be defined. However, restrictions (r1)-(r4) may reduce this freedom degree in some cases. And, indeed:

(a) If $n=1$ then $m=1$ and $u_{N}=u_{1}=u_{1}^{*}=u_{M}^{*}$ is the only parameter.

(b) In this case, $B=B^{N}$, the only union $B_{1}$ is not a singleton, and $u_{1}^{*}=u_{M}^{*}=u_{N}$, which reduces the freedom degree to $n+1$.

(c) Here, $B=B^{n}$ and $u_{M}^{*}=u_{N}$, each union is a singleton with $u_{i}^{*}=u_{i}$ for all $i \in M$, and the freedom degree reduces again to $n+1$.

(d) The number $p$ of singletons satisfies $0 \leq p<m$, since $p=m$ would imply $m=n$. The only restrictions are $u_{k}^{*}=u_{i}$ for each singleton $B_{k}=\{i\}$, so the freedom degree reduces to $n+1+m+1-p$.

In the sequel we will set $d=\operatorname{dim} E_{N}^{B}$.

\subsection{Axiomatic characterization of the modified Shapley rule}

Let us consider the following properties for a coalitional sharing rule $g: E_{N} \longrightarrow \mathbb{R}^{n}$.

(i) Group rationality: $\sum_{i \in N} g_{i}\left[u, B, u^{*}\right]=u_{M}^{*}$ for every $\left[u, B, u^{*}\right]$ in $N$.

(ii) Individual rationality: if $\Delta\left(u^{*}\right) \geq 0$ and $\Delta\left(u^{k}\right) \geq 0$ for all $k \in M$ then $g_{i}\left[u, B, u^{*}\right] \geq u_{i}$ for all $i \in N$.

(iii) Coalitional rationality: if $\Delta\left(u^{*}\right) \geq 0$ then $\sum_{i \in B_{k}} g_{i}\left[u, B, u^{*}\right] \geq u_{k}^{*}$ for all $k \in M$.

(iv) Symmetry: if $u_{i}=u_{j}$ and $i, j \in B_{k}$ for some $k \in M$ then $g_{i}\left[u, B, u^{*}\right]=g_{j}\left[u, B, u^{*}\right]$.

(v) Additivity: for all $B$ and all $\left[u, B, u^{*}\right]$ and $\left[v, B, v^{*}\right]$ in $E_{N}^{B}$,

$$
g\left[u+v, B, u^{*}+v^{*}\right]=g\left[u, B, u^{*}\right]+g\left[v, B, v^{*}\right]
$$

(vi) Singletons: if $B_{k}=\{i\}$ then $g_{i}\left[u, B, u^{*}\right]=\varphi_{k}\left[u^{*}\right]$.

We shall show that this set of properties characterizes the modified Shapley rule $\psi$, defined by Eq. (2). The statement is as follows.

Theorem 8.4 (Theorem 6.3) There is one and only one coalitional sharing rule on $E_{N}$ that satisfies properties (i)-(vi). It is the modified Shapley rule $\psi$.

The proof of this result will be similar to that of Theorem 2.3. The existence part is straightforward using Eq. (2), whereas for the uniqueness part we will need to describe a suitable basis $\mathcal{B}_{0}=\left\{w_{0}^{1}, w_{0}^{2}, \ldots, w_{0}^{d}\right\}$ of $E_{N}^{B}$, where $d=\operatorname{dim} E_{N}^{B}$, for each nontrivial $B$. Each member of this basis will be of the form $w_{0}^{h}=u_{0}^{h} \odot\left(u_{0}^{h}\right)^{*}$, for $1 \leq h \leq d$. 
Example 8.5 (a) Let us proceed to show this basis for a "large enough" numerical example, and to give the rules to construct it in general. In this example, $n=9$ and

$$
B=\{\{1\},\{2\},\{3,4\},\{5,6\},\{7,8,9\}\},
$$

so $m=5, b_{1}=1, b_{2}=1, b_{3}=2, b_{4}=2$ and $b_{5}=3, p=2$, and $\operatorname{dim} E_{N}^{B}=14$. The basis is described in Table 2. The local problems $\left(u_{0}^{h}\right)^{1}$ and $\left(u_{0}^{h}\right)^{2}$ corresponding to the unions that are singletons are trivial and do not appear, but we include the local problems corresponding to the remaining unions, not indispensable to build the basis, because they may well help to fully understand the procedure. All problems are (super)additive.

\begin{tabular}{|c|c|c|c|c|c|}
\hline$w_{0}^{h}$ & $u_{0}^{h}$ & $\left(u_{0}^{h}\right)^{*}$ & $\left(u_{0}^{h}\right)^{3}$ & $\left(u_{0}^{h}\right)^{4}$ & $\left(u_{0}^{h}\right)^{5}$ \\
\hline$w_{0}^{1}$ & $(1,0,0,0,0,0,0,0,0 \mid 1)$ & $(1,0,0,0,0 \mid 1)$ & $(0,0 \mid 0)$ & $(0,0 \mid 0)$ & $(0,0,0 \mid 0)$ \\
$w_{0}^{2}$ & $(0,1,0,0,0,0,0,0,0 \mid 1)$ & $(0,1,0,0,0 \mid 1)$ & $(0,0 \mid 0)$ & $(0,0 \mid 0)$ & $(0,0,0 \mid 0)$ \\
$w_{0}^{3}$ & $(0,0,1,0,0,0,0,0,0 \mid 1)$ & $(0,0,1,0,0 \mid 1)$ & $(1,0 \mid 1)$ & $(0,0 \mid 0)$ & $(0,0,0 \mid 0)$ \\
$w_{0}^{4}$ & $(0,0,0,1,0,0,0,0,0 \mid 1)$ & $(0,0,1,0,0 \mid 1)$ & $(0,1 \mid 1)$ & $(0,0 \mid 0)$ & $(0,0,0 \mid 0)$ \\
$w_{0}^{5}$ & $(0,0,0,0,1,0,0,0,0 \mid 1)$ & $(0,0,0,1,0 \mid 1)$ & $(0,0 \mid 0)$ & $(1,0 \mid 1)$ & $(0,0,0 \mid 0)$ \\
$w_{0}^{6}$ & $(0,0,0,0,0,1,0,0,0 \mid 1)$ & $(0,0,0,1,0 \mid 1)$ & $(0,0 \mid 0)$ & $(0,1 \mid 1)$ & $(0,0,0 \mid 0)$ \\
$w_{0}^{7}$ & $(0,0,0,0,0,0,1,0,0 \mid 1)$ & $(0,0,0,0,1 \mid 1)$ & $(0,0 \mid 0)$ & $(0,0 \mid 0)$ & $(1,0,0 \mid 1)$ \\
$w_{0}^{8}$ & $(0,0,0,0,0,0,0,1,0 \mid 1)$ & $(0,0,0,0,1 \mid 1)$ & $(0,0 \mid 0)$ & $(0,0 \mid 0)$ & $(0,1,0 \mid 1)$ \\
$w_{0}^{9}$ & $(0,0,0,0,0,0,0,0,1 \mid 1)$ & $(0,0,0,0,1 \mid 1)$ & $(0,0 \mid 0)$ & $(0,0 \mid 0)$ & $(0,0,1 \mid 1)$ \\
$w_{0}^{10}$ & $(1,1,1,1,1,1,1,1,1 \mid 10)$ & $(1,1,2,2,3 \mid 9)$ & $(1,1 \mid 2)$ & $(1,1 \mid 2)$ & $(1,1,1 \mid 3)$ \\
\hline$w_{0}^{11}$ & $(0,0,0,0,0,0,0,0,0 \mid 0)$ & $(0,0,1,1,1 \mid 3)$ & $(0,0 \mid 1)$ & $(0,0 \mid 1)$ & $(0,0,0 \mid 1)$ \\
$w_{0}^{12}$ & $(0,0,0,0,0,0,0,0,0 \mid 0)$ & $(0,0,0,1,1 \mid 2)$ & $(0,0 \mid 0)$ & $(0,0 \mid 1)$ & $(0,0,0 \mid 1)$ \\
$w_{0}^{13}$ & $(0,0,0,0,0,0,0,0,0 \mid 0)$ & $(0,0,0,0,1 \mid 1)$ & $(0,0 \mid 0)$ & $(0,0 \mid 0)$ & $(0,0,0 \mid 1)$ \\
$w_{0}^{14}$ & $(0,0,0,0,0,0,0,0,0 \mid 0)$ & $(0,0,0,0,0 \mid 1)$ & $(0,0 \mid 0)$ & $(0,0 \mid 0)$ & $(0,0,0 \mid 0)$ \\
\hline
\end{tabular}

Table 2: The basis $\mathcal{B}_{0}$ of $E_{N}^{B}$ for $n=9$ and $B=\{\{1\},\{2\},\{3,4\},\{5,6\},\{7,8,9\}\}$

The rules for constructing such a basis for arbitrary $n$ and nontrivial $B$ are the following:

- First column $u_{0}^{h}$ :

- from 1 to $n$, the individual utilities form the unit matrix $n \times n$ and $\Delta=0$ (additive problems)

- for $n+1$, all individual utilities equal 1 and $\Delta=1$ (superadditive problem)

- from $n+2$ to $d$, the individual utilities vanish and $\Delta=0$ (additive problems)

- Second column $\left(u_{0}^{h}\right)^{*}$ : 
- from 1 to $n+1$, the individual utility of each union is the sum of the individual utilities of its members in $u_{0}^{h}$ and $\Delta=0$ (additive problems)

- from $n+2$ to $d$, and starting from the bottom $\left(w_{0}^{d}\right)$, we prepare problems where all individual utilities initially vanish but are successively replaced by utility 1 , oneby-one, from the left to the right, and $\Delta=1$ for $w_{0}^{d}$ (superadditive problem) but $\Delta=0$ for all its predecessors (additive problems)

- Next columns: all local problems, derived from the previous rules, are (super)additive

(b) The interested reader is invited to try this procedure for different $n$ and $B$. For example, if $n=4$ and $B=\{\{1,2\},\{3,4\}\}$ the basis $\mathcal{B}_{0}$ is given by Table 3 .

\begin{tabular}{|r|c|c|c|c|}
\hline$w_{0}^{h}$ & $u_{0}^{h}$ & $\left(u_{0}^{h}\right)^{*}$ & $\left(u_{0}^{h}\right)^{1}$ & $\left(u_{0}^{h}\right)^{2}$ \\
\hline$w_{0}^{1}$ & $(1,0,0,0 \mid 1)$ & $(1,0 \mid 1)$ & $(1,0 \mid 1)$ & $(0,0 \mid 0)$ \\
$w_{0}^{2}$ & $(0,1,0,0 \mid 1)$ & $(1,0 \mid 1)$ & $(0,1 \mid 1)$ & $(0,0 \mid 0)$ \\
$w_{0}^{3}$ & $(0,0,1,0 \mid 1)$ & $(0,1 \mid 1)$ & $(0,0 \mid 0)$ & $(1,0 \mid 1)$ \\
$w_{0}^{4}$ & $(0,0,0,1 \mid 1)$ & $(0,1 \mid 1)$ & $(0,0 \mid 0)$ & $(0,1 \mid 1)$ \\
$w_{0}^{5}$ & $(1,1,1,1 \mid 5)$ & $(2,2 \mid 4)$ & $(1,1 \mid 2)$ & $(1,1 \mid 2)$ \\
\hline$w_{0}^{6}$ & $(0,0,0,0 \mid 0)$ & $(1,1 \mid 2)$ & $(0,0 \mid 1)$ & $(0,0 \mid 1)$ \\
$w_{0}^{7}$ & $(0,0,0,0 \mid 0)$ & $(0,1 \mid 1)$ & $(0,0 \mid 0)$ & $(0,0 \mid 1)$ \\
$w_{0}^{8}$ & $(0,0,0,0 \mid 0)$ & $(0,0 \mid 1)$ & $(0,0 \mid 0)$ & $(0,0 \mid 0)$ \\
\hline
\end{tabular}

Table 3: The basis $\mathcal{B}_{0}$ of $E_{N}^{B}$ for $n=4$ and $B=\{\{1,2\},\{3,4\}\}$

$$
\left(\begin{array}{rrrrrrrrrrrrrr}
1 & 0 & 0 & 0 & 0 & 0 & 0 & 0 & 0 & 1 & 0 & 0 & 0 & 1 \\
0 & 1 & 0 & 0 & 0 & 0 & 0 & 0 & 0 & 1 & 0 & 0 & 0 & 1 \\
0 & 0 & 1 & 0 & 0 & 0 & 0 & 0 & 0 & 1 & 1 & 0 & 0 & 1 \\
0 & 0 & 0 & 1 & 0 & 0 & 0 & 0 & 0 & 1 & 1 & 0 & 0 & 1 \\
0 & 0 & 0 & 0 & 1 & 0 & 0 & 0 & 0 & 1 & 0 & 1 & 0 & 1 \\
0 & 0 & 0 & 0 & 0 & 1 & 0 & 0 & 0 & 1 & 0 & 1 & 0 & 1 \\
0 & 0 & 0 & 0 & 0 & 0 & 1 & 0 & 0 & 1 & 0 & 0 & 1 & 1 \\
0 & 0 & 0 & 0 & 0 & 0 & 0 & 1 & 0 & 1 & 0 & 0 & 1 & 1 \\
0 & 0 & 0 & 0 & 0 & 0 & 0 & 0 & 1 & 1 & 0 & 0 & 1 & 1 \\
1 & 1 & 1 & 1 & 1 & 1 & 1 & 1 & 1 & 10 & 2 & 2 & 3 & 9 \\
0 & 0 & 0 & 0 & 0 & 0 & 0 & 0 & 0 & 0 & 1 & 1 & 1 & 3 \\
0 & 0 & 0 & 0 & 0 & 0 & 0 & 0 & 0 & 0 & 0 & 1 & 1 & 2 \\
0 & 0 & 0 & 0 & 0 & 0 & 0 & 0 & 0 & 0 & 0 & 0 & 1 & 1 \\
0 & 0 & 0 & 0 & 0 & 0 & 0 & 0 & 0 & 0 & 0 & 0 & 0 & 1
\end{array}\right)
$$

Matrix of coordinates of the $\mathcal{B}_{0}$ members for $n=9$ 
Lemma 8.6 Assume that a nontrivial coalition structure B has been fixed and ordered in such $a$ way that the singletons (if any) occupy the first places. Then $n>1,1<m<n$ and $d=$ $\operatorname{dim} E_{N}^{B}=n+m-p+2$. A basis $\mathcal{B}_{0}=\left\{w_{0}^{1}, w_{0}^{2}, \ldots, w_{0}^{d}\right\}$ of $E_{N}^{B}$, where all problems involved for each member of $\mathcal{B}_{0}$ are (super)additive, can be obtained as indicated in Example 8.5(a).

Proof. In Example 8.5(a), since $\left(u_{0}^{h}\right)_{1}^{*}$ and $\left(u_{0}^{h}\right)_{2}^{*}$ are imposed by restriction (r4) and hence they cannot be chosen, the parameters that define each $w_{0}^{h}$ are only

$$
\left(u_{0}^{h}\right)_{1}, \quad\left(u_{0}^{h}\right)_{2}, \quad \ldots, \quad\left(u_{0}^{h}\right)_{9}, \quad\left(u_{0}^{h}\right)_{N}, \quad\left(u_{0}^{h}\right)_{3}^{*}, \quad\left(u_{0}^{h}\right)_{4}^{*}, \quad\left(u_{0}^{h}\right)_{5}^{*}, \quad\left(u_{0}^{h}\right)_{M}^{*} .
$$

Eq. (4) provides the matrix defined by these parameters, the rows of which represent the coordinates of each $w_{0}^{h}$ in an obvious "canonical basis" of $E_{N}^{B}$-which is not suitable to our interests because most of its members are not (super)additive.

It is easy to see that this is a regular matrix, so $\left\{w_{0}^{h}: h=1, \ldots, 14\right\}$ is a linearly independent set and therefore a basis of $E_{N}^{B}$. Indeed, by subtracting rows 1 to 9 from row 10 we obtain an equivalent matrix (in rank terms) of the form

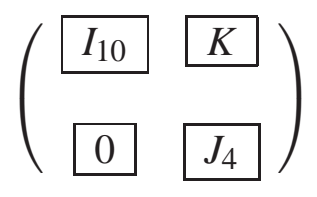

where $I_{10}$ is the unit submatrix $10 \times 10, J_{4}$ is a triangular regular submatrix $4 \times 4,0$ represents the null submatrix $4 \times 10$, and $K$ is a $10 \times 4$ submatrix that does not matter to see that the rank of the entire matrix is 14 . Of course, mutatis mutandis the argument also holds for Example 8.5(b) and remains clearly valid for any $n$ and any nontrivial coalition structure $B$.

Remark 8.7 Moreover, there is a nice linear expression in terms of $\mathcal{B}_{0}$ of any CS-problem $w=$ $u \odot u^{*}$, with $B$ as coalition structure, but at this point our assertion is a well founded conjecture only. It can be checked in every particular case, using e.g. MATHEMATICA, but we have no general proof. Anyway, this is not necessary for the proof of the characterization theorem.

Thus, we have: in Example 8.5(a),

$$
\begin{aligned}
& w=u \odot u^{*}=\left[u_{1}-\Delta(u)\right] w_{0}^{1}+\left[u_{2}-\Delta(u)\right] w_{0}^{2}+\left[u_{3}-\Delta(u)\right] w_{0}^{3}+\left[u_{4}-\Delta(u)\right] w_{0}^{4}+ \\
& {\left[u_{5}-\Delta(u)\right] w_{0}^{5}+\left[u_{6}-\Delta(u)\right] w_{0}^{6}+\left[u_{7}-\Delta(u)\right] w_{0}^{7}+\left[u_{8}-\Delta(u)\right] w_{0}^{8}+\left[u_{9}-\Delta(u)\right] w_{0}^{9}+} \\
& \Delta(u) w_{0}^{10}+\Delta\left(u^{3}\right) w_{0}^{11}+\left[\Delta\left(u^{4}\right)-\Delta\left(u^{3}\right)\right] w_{0}^{12}+\left[\Delta\left(u^{5}\right)-\Delta\left(u^{4}\right)\right] w_{0}^{13}+\Delta\left(u^{*}\right) w_{0}^{14},
\end{aligned}
$$

and, in Example 8.5(b),

$$
\begin{aligned}
& w=u \odot u^{*}=\left[u_{1}-\Delta(u)\right] w_{0}^{1}+\left[u_{2}-\Delta(u)\right] w_{0}^{2}+\left[u_{3}-\Delta(u)\right] w_{0}^{3}+\left[u_{4}-\Delta(u)\right] w_{0}^{4}+ \\
& \Delta(u) w_{0}^{5}+\Delta\left(u^{1}\right) w_{0}^{6}+\left[\Delta\left(u^{2}\right)-\Delta\left(u^{1}\right)\right] w_{0}^{7}+\Delta\left(u^{*}\right) w_{0}^{8}
\end{aligned}
$$

The general rule for the coefficients of such a linear combination is as follows:

- for $h=1, \ldots, n$, the coefficient of $w_{0}^{h}$ is $u_{h}-\Delta(u)$

- the coefficient of $w_{0}^{n+1}$ is $\Delta(u)$ 
- for $h=n+2, \ldots, d$, if $B_{k}$ is the first union that is not a singleton, the coefficients are: $\Delta\left(u^{k}\right)$ for $w_{0}^{n+2}, \Delta\left(u^{k+1}\right)-\Delta\left(u^{k}\right)$ for $w_{0}^{n+3}, \Delta\left(u^{k+2}\right)-\Delta\left(u^{k+1}\right)$ for $w_{0}^{n+4}$, and so on, but the last one, for $w_{0}^{d}$, is simply $\Delta\left(u^{*}\right)$

The regularity of these expressions confirms the suitability of the basis $\mathcal{B}_{0}$.

Proof of Theorem 8.8 (Theorem 6.3) (Existence) It suffices to check that the modified Shapley rule $\psi$ satisfies (i)-(vi), and this will follow at once from Eq. (2).

(i) Group rationality. We have

$$
\begin{aligned}
\sum_{i \in N} \psi_{i}\left[u, B, u^{*}\right] & =\sum_{i \in N} u_{i}+\sum_{k \in M} \sum_{i \in B_{k}}\left[\frac{\Delta\left(u^{k}\right)}{b_{k}}+\frac{\Delta\left(u^{*}\right)}{m b_{k}}\right]=\sum_{k \in M}\left[\sum_{i \in B_{k}} u_{i}+\Delta\left(u^{k}\right)\right]+\Delta\left(u^{*}\right)= \\
\sum_{k \in M} u_{k}^{*}+\Delta\left(u^{*}\right) & =u_{M}^{*}
\end{aligned}
$$

(ii) Individual rationality. It is clear that if $\Delta\left(u^{*}\right) \geq 0$ and $\Delta\left(u^{k}\right) \geq 0$ for all $k \in M$ then $\psi_{i}\left[u, B, u^{*}\right] \geq u_{i}$ for all $i \in N$.

(iii) Coalitional rationality. If $\Delta\left(u^{*}\right) \geq 0$ then, for each $k \in M$,

$$
\sum_{i \in B_{k}} \psi_{i}\left[u, B, u^{*}\right]=\sum_{i \in B_{k}} u_{i}+\Delta\left(u^{k}\right)+\frac{\Delta\left(u^{*}\right)}{m}=u_{k}^{*}+\frac{\Delta\left(u^{*}\right)}{m} \geq u_{k}^{*}
$$

(iv) Symmetry. Since the second and third terms of Eq. (2) are constant in each $B_{k}$, if $u_{i}=u_{j}$ and $i, j \in B_{k}$ for some $k \in M$ then $\psi_{i}\left[u, B, u^{*}\right]=\psi_{j}\left[u, B, u^{*}\right]$.

(v) Additivity. This property is clearly satisfied by the linearity of Eq. (2) with respect to $u, u^{*}$ and all $u^{k}$.

(vi) Singletons. This is a particular case of Proposition 6.2(b).

(Uniqueness) We shall see that, for every coalition structure $B$, if $g$ satisfies (i)-(vi) on $E_{N}^{B}$ then $g$ is uniquely determined on $E_{N}^{B}$. Thus, we will have $g=\psi$ in each $E_{N}^{B}$ and hence in $E_{N}$.

1. If $B=B^{n}$ then only $u$ matters since $M=N$ and $u^{*}=u$. Properties (i), (ii), (iv) and (v) become the properties of the Shapley rule $\varphi$ and, according to Theorem 2.3 and Proposition 6.2(a), it follows that $g\left[u, B^{n}, u^{*}\right]=\varphi[u]=\psi\left[u, B^{n}, u^{*}\right]$.

2. Similarly, if $B=B^{N}$ then only $u$ matters since $B_{1}=N, u^{*}$ is trivial and $u^{1}=u$. Again, properties (i), (ii), (iv) and (v) become the properties of the Shapley rule $\varphi$ and, according to Theorem 2.3 and Proposition 6.2(a), it follows that $g\left[u, B^{N}, u^{*}\right]=\varphi[u]=\psi\left[u, B^{N}, u^{*}\right]$.

3. Therefore, in the sequel we can assume that $B$ is not trivial. We will use the basis

$$
\mathcal{B}_{0}=\left\{w_{0}^{1}, w_{0}^{2}, \ldots, w_{0}^{d}\right\} \quad\left(d=\operatorname{dim} E_{N}^{B}\right) .
$$

whose existence has been proven in Lemma 8.6. Since all problems involved in this basis are (super)additive by construction, it is straightforward to check that properties (i), (ii), (iii), (iv) and (vi) univocally determine the action of $g$ on each member of $\mathcal{B}_{0}$. 
Now, using property (v), we will prove that $g$ is univocally determined also on any CS-problem $w \in E_{N}^{B}$. First, we note that, for any $\lambda \geq 0$ and $h=1,2, \ldots, d, g$ is also univocally determined on any vector of the form $\lambda w_{0}^{h}$, still (super)additive, and $g\left[\lambda w_{0}^{h}\right]=\lambda g\left[w_{0}^{h}\right]$. Let us assume that

$$
w=\sum_{h=1}^{d} \lambda_{h} w_{0}^{h}
$$

Even if $w$ is (super)additive, some $\lambda<0$ might appear in this expressions. Then we write

$$
w+\sum_{\lambda_{h}<0}\left(-\lambda_{h}\right) w_{0}^{h}=\sum_{\lambda_{h}>0} \lambda_{h} w_{0}^{h}
$$

where three vectors appear that are defined by (super)additive problems. Then we can apply property (v) to obtain

$$
g[w]+\sum_{\lambda_{h}<0}\left(-\lambda_{h}\right) g\left[w_{0}^{h}\right]=\sum_{\lambda_{h}>0} \lambda_{h} g\left[w_{0}^{h}\right]
$$

that finally gives

$$
g[w]=\sum_{h=1}^{d} \lambda_{h} g\left[w_{0}^{h}\right] .
$$

Summing up, $g$ is univocally determined on $E_{N}^{B}$ and hence $g=\psi$ on this vector space.

As to the logical independence of the axiomatic system described above, first we solve some particular and quite trivial cases:

- If $n=1$ then group rationality (i) suffices to characterize $\psi$.

- If $n=2$ then $B^{n}$ and $B^{N}$ are the only coalition structures. Properties (i), (ii), (iv) and (v) suffice to characterize $\psi$ and are, therefore, logically independent. In fact, $\psi\left[u, B, u^{*}\right]$ and $\varphi[u]$ coincide (cf. Proposition 6.2 or the uniqueness part in the proof of Theorem 6.3) and these four properties reduce to the axioms of the Shapley rule used in Theorem 2.3.

- If $n=3$ then (i), (ii), (iv), (v) and (vi) suffice to characterize $\psi$ and are logically independent.

Then, in the sequel we can assume $n \geq 4$. In this general case we show that the six properties are logically independent. To this end, it suffices to find six rules that satisfy all axioms but one. Only a CS-problem that shows the failure is needed in each case (a counterexample). All counterexamples given below concern $n=4$ agents and are based on (super)additive problems, and all of them might be extended to any number $n$ of agents by adding $n-4$ null agents.

- A rule that fails to satisfy (i) Group rationality.

$$
g_{i}\left[u, B, u^{*}\right]= \begin{cases}\varphi_{k}\left[u^{*}\right] & \text { if } B_{k}=\{i\} \text { is a singleton } \\ u_{i}+\frac{\Delta\left(u^{k}\right)}{b_{k}} & \text { otherwise }\end{cases}
$$

It is easy to verify that $g$ satisfies (ii), (iii), (iv), (v) and (vi). 
Instead, for $u=(1,1,2,3 \mid 8), B=\{\{1,2\},\{3,4\}\}$, and $u^{*}=(4,7 \mid 15)$ we find that

$$
\sum_{i \in N} g_{i}\left[u, B, u^{*}\right]=11 \neq 15=u_{M}^{*}
$$

- A rule that fails to satisfy (ii) Individual rationality.

$$
g_{i}\left[u, B, u^{*}\right]=\frac{\varphi_{k}\left[u^{*}\right]}{b_{k}} \quad \text { if } i \in B_{k}
$$

It is easy to verify that $g$ satisfies (i), (iii), (iv), (v) and (vi).

Instead, for $u=(1,3,2,5 \mid 12), B=\{\{1,2\},\{3,4\}\}$, and $u^{*}=(4,8 \mid 12)$ we find that

$$
g_{2}\left[u, B, u^{*}\right]=2 \nsupseteq 3=u_{2} .
$$

- A rule that fails to satisfy (iii) Coalitional rationality.

We will say that $B$ is wide if $m \geq 2$, and $b_{1}, b_{2}>1$.

$$
g_{i}\left[u, B, u^{*}\right]= \begin{cases}u_{i}+\frac{\Delta\left(u^{*}\right)}{m b_{1}} & \text { if } B \text { is wide and } i \in B_{1} \\ u_{i}+\frac{\Delta\left(u^{1}\right)}{b_{2}}+\frac{\Delta\left(u^{2}\right)}{b_{2}}+\frac{\Delta\left(u^{*}\right)}{m b_{2}} & \text { if } B \text { is wide and } i \in B_{2} \\ \psi_{i}\left[u, B, u^{*}\right] & \text { otherwise, for this and any other } B\end{cases}
$$

It is easy to verify that $g$ satisfies (i), (ii), (iv), (v) and (vi).

Instead, for $u=(1,2,2,3 \mid 9), B=\{\{1,2\},\{3,4\}\}$, and $u^{*}=(9,7 \mid 20)$ we find that

$$
\sum_{i \in B_{1}} g_{i}\left[u, B, u^{*}\right]=5 \nsupseteq 9=u_{1}^{*} .
$$

- A rule that fails to satisfy (iv) Symmetry.

We assume that the natural ordering of $N$ is preserved within each $B_{k}$ for any $B$. We will call the leader of $B_{k}$ to the first member of $B_{k}$ according to this induced ordering.

$$
g_{i}\left[u, B, u^{*}\right]= \begin{cases}u_{i}+\varphi_{k}\left[u^{*}\right]-\sum_{j \in B_{k}} u_{j} & \text { if } i \text { is the leader of } B_{k} \\ u_{i} & \text { otherwise }\end{cases}
$$

It is easy to verify that $g$ satisfies (i), (ii), (iii), (v) and (vi).

Instead, for $u=(1,1,2,2 \mid 7), B=\{\{1,2\},\{3,4\}\}$, and $u^{*}=(4,6 \mid 14)$ we find that

$$
u_{1}=u_{2} \quad \text { but } \quad g_{1}\left[u, B, u^{*}\right]=5 \neq 1=g_{2}\left[u, B, u^{*}\right]
$$


- A rule that fails to satisfy (v) Additivity. It combines proportional rule and Shapley rule.

$$
g_{i}\left[u, B, u^{*}\right]= \begin{cases}\frac{u_{i}}{\sum_{j \in B_{k}} u_{j}} \varphi_{k}\left[u^{*}\right] & \text { if } i \in B_{k} \text { such that } u_{j}>0 \text { for all } j \in B_{k} \\ \psi_{i}\left[u, B, u^{*}\right] & \text { otherwise, for this and any other } B\end{cases}
$$

It is easy to verify that $g$ satisfies (i), (ii), (iii), (iv) and (vi).

Instead, if

$$
\begin{array}{lll}
u=(1,1,0,3 \mid 6), & B=\{\{1,2\},\{3,4\}\}, & u^{*}=(4,8 \mid 20) \\
v=(4,0,1,1 \mid 7), & B=\{\{1,2\},\{3,4\}\}, & v^{*}=(8,4 \mid 20)
\end{array}
$$

then

$$
u+v=(5,1,1,4 \mid 13), \quad B=\{\{1,2\},\{3,4\}\}, \quad u^{*}+v^{*}=(12,12 \mid 40)
$$

and

$$
g\left[u+v, B, u^{*}+v^{*}\right]=(16.67,3.33,4,16) \neq(4,4,4,8)+(8,4,4,4)=g\left[u, B, u^{*}\right]+g\left[v, B, v^{*}\right] .
$$

- A rule that fails to satisfy (vi) Singletons.

$$
g_{i}\left[u, B, u^{*}\right]=u_{i}+\frac{\Delta\left(u^{k}\right)}{b_{k}}+\frac{\Delta\left(u^{*}\right)}{n} \quad \text { if } i \in B_{k}
$$

It is easy to verify that $g$ satisfies (i), (ii), (iii), (iv) and (v).

Instead, for $u=(1,1,2,3 \mid 8), B=\{\{1\},\{2,3,4\}\}$, and $u^{*}=(1,9 \mid 14)$ we find that $B_{1}$ is a singleton but

$$
g_{1}\left[u, B, u^{*}\right]=2 \neq 3=\varphi_{1}\left[u^{*}\right] .
$$

Theorem 8.8 (Theorem 6.10) There is one and only one coalitional sharing rule defined on the cone $\left(E_{N}^{B}\right)^{*}$ that satisfies properties $(i)-(v i)$ in this cone. It is (the restriction of) the modified Shapley rule $\psi$.

Proof. (Existence) The proof is exactly the same as in Theorem 6.3.

(Uniqueness) The proof is the same as in Theorem 6.3 provided that we slightly modify the basis $\mathcal{B}_{0}$ introduced in Example 8.5(a) and Lemma 8.6 and replace it by a new basis $\overline{\mathcal{B}}_{0}$.

For each fixed and nontrivial coalition structure $B$, all members of $\mathcal{B}_{0}$ were CS-problems of the form $w=u \odot u^{*}$ where $u, u^{*}$ and all $u^{k}$ were (super)additive. However, this does not ensure that all of them belong to the cone $\left(E_{N}^{B}\right)^{*}$. Hence a little modification is needed in $\mathcal{B}_{0}$.

The new basis $\overline{\mathcal{B}}_{0}$ derives from $\mathcal{B}_{0}$ by only replacing, just for $h=1,2, \ldots, n, n+1$, the total utility $\left(u_{0}^{h}\right)_{M}^{*}$ by $m\left(u_{0}^{h}\right)_{N}$. Then we obtain a new set $\overline{\mathcal{B}}_{0}=\left\{\bar{w}_{0}{ }^{1}, \bar{w}_{0}{ }^{2}, \ldots, \bar{w}_{0}{ }^{d}\right\}$, the matrix of which is still regular as the matrix given in Eq. (4), so $\overline{\mathcal{B}}_{0}$ is also a basis of $\left(E_{N}^{B}\right)$.

However, now, for each member $\bar{w}=\bar{u} \odot \bar{u}^{*}$ of $\overline{\mathcal{B}}_{0}$, we have

(1) $\Delta\left(\bar{u}^{*}\right)>0$

(2) $\Delta\left(\bar{u}^{k}\right) \geq 0$ for all $k$, and 
(3) $\Delta\left(\bar{u}^{*}\right)=m \Delta(\bar{u})>\frac{m b_{k}}{n} \Delta(\bar{u})$ for all $k$,

which are the sufficient conditions stated in Example 6.6 for belonging to $\left(E_{N}^{B}\right)^{*}$. Hence, since all members of the new basis belong to the cone, the uniqueness proof follows the same pattern as in Theorem 6.3. 